\title{
THE DOUBLE AND DUAL OF A QUASITRIANGULAR LIE BIALGEBRA
}

\author{
Timothy J. Hodges* AND Milen Yakimov ${ }^{\dagger}$
}

\begin{abstract}
Let $G$ be a connected, simply connected Poisson-Lie group with quasitriangular Lie bialgebra $\mathfrak{g}$. An explicit description of the double $\mathcal{D}(\mathfrak{g})$ is given, together with the embeddings of $\mathfrak{g}$ and $\mathfrak{g}^{*}$. This description is then used to provide a construction of the double $\mathcal{D}(G)$. The aim of this work is to describe $\mathcal{D}(G)$ in sufficient detail to be able to apply the procedures of Semenov-Tian-Shansky and Drinfeld for the classification of symplectic leaves and Poisson homogeneous spaces for Poisson-Lie groups.
\end{abstract}

\section{Introduction}

Knowing explicitly the Lie algebra structure of the double of a Lie bialgebra is necessary for the classification of the Poisson homogeneous spaces and symplectic leaves of the corresponding Poisson-Lie groups.

To be more precise, let $\mathfrak{g}$ be a Lie bialgebra over a field $k$ and $\mathcal{D}(\mathfrak{g})$ be its double. By the dual Lie bialgebra $\mathfrak{g}^{*}$ of $\mathfrak{g}$, we will mean the standard dual Lie bialgebra of $\mathfrak{g}$, equipped with opposite cobracket. When $k=\mathbb{R}$ or $\mathbb{C}$ the connected, simply connected Poisson-Lie groups with tangent Lie bialgebras $\mathfrak{g}$, $\mathfrak{g}^{*}$ and $\mathcal{D}(\mathfrak{g})$ will be denoted by $G, G^{*}$, and $\mathcal{D}(G) . \mathcal{D}(G)$ and $G^{*}$ are called the double and dual Poisson-Lie groups of $G$, respectively. Let $j: G \rightarrow \mathcal{D}(G)$ and $j^{*}: G^{*} \rightarrow \mathcal{D}(G)$ be the group homomorphisms corresponding to the embeddings $\mathfrak{g} \subset \mathcal{D}(\mathfrak{g})$ and $\mathfrak{g}^{*} \subset \mathcal{D}(\mathfrak{g})$.

According to a result of Drinfeld [3], the Poisson homogeneous spaces for $G$ with connected stabilizers are in one to one correspondence with the orbits of the adjoint action of $G$ on the variety of the Lagrangian subalgebras $\mathfrak{l}$ of $\mathcal{D}(\mathfrak{g})$ for which $\mathfrak{l} \cap \mathfrak{g}$ integrates to a closed subgroup of $G$.

By a theorem of Semenov-Tian-Shansky [11], the symplectic leaves of $G$ are the orbits of the (local) dressing action of $G^{*}$ on $G$ which is the lifting under $G \rightarrow \mathcal{D}(G) / j^{*}\left(G^{*}\right)$ of the left action of $G^{*}$ on $\mathcal{D}(G) / j^{*}\left(G^{*}\right)$. Again to work with it in a concrete situation, one needs an explicit description of $\mathcal{D}(G)$ and of the images of $G$ and $G^{*}$. For $G$ semisimple, the symplectic leaves of $G$ were described using this procedure by Hodges and Levasseur [6] for the standard

Received August 17, 2000.

*The author was partially supported by grants from the National Security Agency and the Charles P. Taft Foundation.

${ }^{\dagger}$ The author was supported by NSF grants DMS94-00097 and DMS96-03239. 
bialgebra structure and by Yakimov [14] for the structures associated to a general Belavin-Drinfeld $r$-matrix.

If the Lie bialgebra $\mathfrak{g}$ is quasitriangular with an $r$-matrix $r$, the structure of $\mathcal{D}(\mathfrak{g})$ is encoded in the structure of the Lie algebra $\mathfrak{g}$ and the $r$-matrix. For a factorizable Lie bialgebra it was shown by Reshetikhin and Semenov-TianShansky [9] that $\mathcal{D}(\mathfrak{g}) \cong \mathfrak{g} \oplus \mathfrak{g}$. Stolin [12] and Levendorskii, Soibelman [7] showed for triangular structures on complex simple Lie algebras and their compact real forms that $\mathcal{D}(\mathfrak{g}) \cong \mathfrak{g} \ltimes \mathfrak{g}^{*}$. These results enable one to construct $\mathcal{D}(G)$ as $G \times G$ and $G \ltimes \mathfrak{g}^{*}$ respectively. The goal of this note is to extend these results to the general case; that is, to give an explicit description of $\mathcal{D}(\mathfrak{g})$ and of the embeddings $\mathfrak{g}, \mathfrak{g}^{*} \subset \mathcal{D}(\mathfrak{g})$ in terms of the r-matrix and then to lift this description to a construction of $\mathcal{D}(G)$.

Next we briefly explain our results. The subspace $\mathfrak{f}$ of $\mathfrak{g}$ spanned by all components of the tensor $r+r^{21} \in S^{2}(\mathfrak{g})$ is an ideal of $\mathfrak{g}$. In Section 3 we show that $\mathcal{D}(\mathfrak{g})$ is an extension of the semidirect sum $\mathfrak{g} \ltimes \mathfrak{f}$ by an abelian Lie algebra. The 2-cocycle $\alpha$ defining the extension is computed in Section 4 . Section 5 deals with the image of $\mathfrak{g}^{*} \subset \mathcal{D}(\mathfrak{g})$ under this isomorphism. It is described in terms of an explicit 1-cochain for a subalgebra $\mathfrak{b}$ of $\mathfrak{g} \ltimes \mathfrak{f}$ whose coboundary is essentially the restriction of $\alpha$ to $\mathfrak{b}$. In Section 6 we lift this construction to a construction of $\mathcal{D}(G)$ as an extension of $G \ltimes F$ by $\mathfrak{f}^{\perp}$, generalizing the above descriptions of $\mathcal{D}(G)$ in the factorizable and triangular cases. In Section 7 we specialize the results to the two limiting cases of triangular and factorizable structures, i.e. $r+r^{21}=0$ or of full rank.

No assumptions will be made on the ground field $k$ and unless otherwised specified all Lie bialgebras can be infinite dimensional. For a vector space $V$ over $k$, the natural pairing between $\xi \in V^{*}$ and $v \in V$ will be denoted both by $\xi(v)$ and $\langle\xi, v\rangle$. The symbol $\ltimes$ will be used for both semidirect sums and products of Lie algebras and Lie groups.

\section{Lie bialgebras}

Recall that a Lie bialgebra $\mathfrak{g}$ with Lie bracket [., .] and Lie cobracket $\delta$ is called quasitriangular if there exists an element $r \in \mathfrak{g} \otimes \mathfrak{g}$ such that

$$
\delta(x)=\left(\operatorname{ad}_{x} \otimes \mathrm{id}+\mathrm{id} \otimes \operatorname{ad}_{x}\right) r, \forall x \in \mathfrak{g}
$$

and $r$ satisfies the Yang-Baxter equation

$$
\left[r_{12}, r_{13}\right]+\left[r_{12}, r_{23}\right]+\left[r_{13}, r_{23}\right]=0
$$

Let $\mathfrak{g}^{*}$ be the dual Lie bialgebra of $\mathfrak{g}$ with opposite cobracket as explained in the introduction. (This convention is not essential since in Sections 3-7 we only work with the Lie algebra structure of $\mathfrak{g}, \mathfrak{g}^{*}$, and $\mathcal{D}(\mathfrak{g})$.) There are two natural Lie bialgebra homomorphisms $r_{ \pm}: \mathfrak{g}^{*} \rightarrow \mathfrak{g}$ (see [9]), given by

$$
\begin{aligned}
& r_{+}(\xi)=(\xi \otimes \mathrm{id}) r, \\
& r_{-}(\xi)=-(\mathrm{id} \otimes \xi) r, \xi \in \mathfrak{g}^{*} .
\end{aligned}
$$


Recall also that the double Lie bialgebra $\mathcal{D}(\mathfrak{g})$ of $\mathfrak{g}$ is characterized by the properties

1. $\mathcal{D}(\mathfrak{g}) \cong \mathfrak{g} \oplus \mathfrak{g}^{*}$ as a coalgebra,

2. $\mathfrak{g}$ and $\mathfrak{g}^{*}$ are subalgebras of $\mathcal{D}(\mathfrak{g})$ and two elements $x \in \mathfrak{g} \subset \mathcal{D}(\mathfrak{g})$ and $\xi \in \mathfrak{g}^{*} \subset \mathcal{D}(\mathfrak{g})$ commute as

$$
[x, \xi]=\operatorname{ad}_{x}^{*}(\xi)-\operatorname{ad}_{\xi}^{*}(x)
$$

where $a^{*}$ denotes the coadjoint actions of both $\mathfrak{g}$ and $\mathfrak{g}^{*}$.

There is a canonical nondegenerate invariant bilinear form on $\mathcal{D}(\mathfrak{g})$ given by

$$
\left\langle\left\langle x_{1}+\xi_{1}, x_{2}+\xi_{2}\right\rangle\right\rangle:=\left\langle\xi_{1}, x_{2}\right\rangle+\left\langle\xi_{2}, x_{1}\right\rangle, \forall x_{i} \in \mathfrak{g}, \xi_{i} \in \mathfrak{g}^{*}
$$

with respect to which $\left(\mathcal{D}(\mathfrak{g}), \mathfrak{g}, \mathfrak{g}^{*}\right)$ is a Manin triple. For details we refer to [2].

\section{An exact sequence}

Let $\mathfrak{f} \subset \mathfrak{g}$ denote the span of all components of the tensor

$$
\Omega=r+r_{21} \in S^{2}(\mathfrak{g}) .
$$

Since $\delta$ is skewsymmetric, $\Omega$ is $\operatorname{ad}_{\mathfrak{g}}$ invariant. This implies that $\mathfrak{f}$ is an ideal of $\mathfrak{g}$. Denote by $\mathfrak{f}^{\perp}$ the subspace of $\mathfrak{g}^{*}$ orthogonal to $\mathfrak{f} \subset \mathfrak{g}$ :

$$
\mathfrak{f}^{\perp}=\left\{\eta \in \mathfrak{g}^{*} \mid\langle\eta, y\rangle=0, \forall y \in \mathfrak{f}\right\} .
$$

In terms of the maps $r_{ \pm}, \mathfrak{f}$ and $\mathfrak{f}^{\perp}$ are given by

$$
\begin{aligned}
& \mathfrak{f}=\operatorname{Im}\left(r_{+}-r_{-}\right), \\
& \mathfrak{f}^{\perp}=\operatorname{Ker}\left(r_{+}-r_{-}\right) .
\end{aligned}
$$

Define two linear maps

$$
p_{ \pm}: \mathcal{D}(\mathfrak{g}) \rightarrow \mathfrak{g}
$$

by

$$
p_{ \pm}(x+\xi)=x+r_{ \pm}(\xi) ; x \in \mathfrak{g} \subset \mathcal{D}(\mathfrak{g}), \xi \in \mathfrak{g}^{*} \subset \mathcal{D}(\mathfrak{g}) .
$$

The following result is due to Majid [8, 8.2.7]; we include a proof for completeness.

Proposition 3.1. The maps $p_{ \pm}: \mathcal{D}(\mathfrak{g}) \rightarrow \mathfrak{g}$ are homomorphisms of Lie algebras.

In fact the maps $p_{ \pm}$are homomorphisms of Lie bialgebras since $\mathcal{D}(\mathfrak{g}) \cong \mathfrak{g} \oplus \mathfrak{g}^{*}$ as a coalgebra, but we will not need this fact here. fact.

In the proof of this proposition and below we will use the following simple

Lemma 3.2. For all $\xi \in \mathfrak{g}^{*}$ and $x \in \mathfrak{g}$

$$
\begin{aligned}
\operatorname{ad}_{\xi}^{*}(x) & =-(\xi \otimes \mathrm{id})\left(\left(\operatorname{ad}_{x} \otimes \mathrm{id}+\mathrm{id} \otimes \operatorname{ad}_{x}\right) r\right) \\
& =(\mathrm{id} \otimes \xi)\left(\left(\operatorname{ad}_{x} \otimes \mathrm{id}+\mathrm{id} \otimes \operatorname{ad}_{x}\right) r\right) .
\end{aligned}
$$


Proof. For an arbitrary $\xi_{0} \in \mathfrak{g}^{*}$

$$
\begin{aligned}
& \left\langle\operatorname{ad}_{\xi}^{*}(x), \xi_{0}\right\rangle=-\left\langle x,\left[\xi, \xi_{0}\right]\right\rangle \\
& =-\left\langle\delta(x), \xi \otimes \xi_{0}\right\rangle=\left\langle-(\xi \otimes \mathrm{id})\left(\left(\operatorname{ad}_{x} \otimes \mathrm{id}+\mathrm{id} \otimes \operatorname{ad}_{x}\right) r\right), \xi_{0}\right\rangle .
\end{aligned}
$$

Proof of Proposition 3.1. The restrictions of $p_{ \pm}$to $\mathfrak{g} \subset \mathcal{D}(\mathfrak{g})$ and $\mathfrak{g}^{*} \subset \mathcal{D}(\mathfrak{g})$ coincide with the identity and $r_{ \pm}$respectively and thus are homomorphisms. We are left with checking that

$$
\left[p_{ \pm}(x), p_{ \pm}(\xi)\right]=p_{ \pm}([x, \xi])
$$

for $x \in \mathfrak{g} \subset \mathcal{D}(\mathfrak{g}), \xi \in \mathfrak{g}^{*} \subset \mathcal{D}(\mathfrak{g})$. Using Lemma 3.2, for the map $p_{+}$one computes

$$
\begin{aligned}
p_{+}([x, \xi]) & =p_{+}\left(\operatorname{ad}_{x}^{*}(\xi)-\operatorname{ad}_{\xi}^{*}(x)\right) \\
& =\left(\operatorname{ad}_{x}^{*}(\xi) \otimes \mathrm{id}\right) r-\operatorname{ad}_{\xi}^{*}(x) \\
& =-(\xi \otimes \mathrm{id})\left(\left(\operatorname{ad}_{x} \otimes \mathrm{id}\right) r\right)+(\xi \otimes \mathrm{id})\left(\left(\operatorname{ad}_{x} \otimes \mathrm{id}+\mathrm{id} \otimes \operatorname{ad}_{x}\right) r\right) \\
& =\left[x, r_{+}(\xi)\right]=\left[p_{+}(x), p_{+}(\xi)\right]
\end{aligned}
$$

Composing with the natural embeddings of $\mathfrak{g}$ and $\mathfrak{g}^{*}$ into $\mathcal{D}(\mathfrak{g})$, we may consider id and $r_{ \pm}$as maps from $\mathfrak{g}^{*}$ to $\mathcal{D}(\mathfrak{g})$. Thus id $-r_{ \pm}$are also maps from $\mathfrak{g}^{*}$ to $\mathcal{D}(\mathfrak{g})$ and the kernels of $p_{ \pm}$are precisely the images of these maps; that is,

$$
\text { Ker } p_{ \pm}=\left(\mathrm{id}-r_{ \pm}\right) \mathfrak{g}^{*}
$$

Lemma 3.3. The algebras $\operatorname{Ker} p_{+}$and $\operatorname{Ker} p_{-}$mutually commute in $\mathcal{D}(\mathfrak{g})$.

Proof. Since Ker $p_{ \pm}$are ideals of $\mathcal{D}(\mathfrak{g})$, for any $\xi_{1}, \xi_{2} \in \mathfrak{g}^{*}$

$$
\left[\left(\mathrm{id}-r_{+}\right) \xi_{1},\left(\mathrm{id}-r_{-}\right) \xi_{2}\right]=\left(\mathrm{id}-r_{+}\right) \xi
$$

for some $\xi \in \mathfrak{g}^{*}$. Comparing the components in $\mathfrak{g}^{*} \subset \mathcal{D}(\mathfrak{g})$ of the two sides of this equality, we get

$$
\xi=\left[\xi_{1}, \xi_{2}\right]-\operatorname{ad}_{r_{+}\left(\xi_{1}\right)}^{*}\left(\xi_{2}\right)+\operatorname{ad}_{r_{-}\left(\xi_{2}\right)}^{*}\left(\xi_{1}\right) .
$$

Thus Lemma 3.3 is equivalent to the vanishing of the above expression for all $\xi_{1}, \xi_{2} \in \mathfrak{g}^{*}$. For an arbitrary $x \in \mathfrak{g}$

$$
\begin{aligned}
& \left\langle\left[\xi_{1}, \xi_{2}\right]-\operatorname{ad}_{r_{+}\left(\xi_{1}\right)}^{*}\left(\xi_{2}\right)+\operatorname{ad}_{r_{-}\left(\xi_{2}\right)}^{*}\left(\xi_{1}\right), x\right\rangle \\
& =\left\langle\xi_{1} \otimes \xi_{2}, \delta(x)\right\rangle+\left\langle\xi_{2}, \operatorname{ad}_{r_{+}\left(\xi_{1}\right)}(x)\right\rangle-\left\langle\xi_{1}, \operatorname{ad}_{r_{-}\left(\xi_{2}\right)}(x)\right\rangle \\
& =\left\langle\xi_{1} \otimes \xi_{2}, \delta(x)-\left(\mathrm{id} \otimes \operatorname{ad}_{x}\right) r-\left(\operatorname{ad}_{x} \otimes \mathrm{id}\right) r\right\rangle=0 .
\end{aligned}
$$

Combine the maps $p_{ \pm}$into the single morphism

$$
p: \mathcal{D}(\mathfrak{g}) \rightarrow \mathfrak{g} \oplus \mathfrak{g}, p(d)=\left(p_{+}(d), p_{-}(d)\right) .
$$


Using (3.3), for its image we obtain

$$
\begin{aligned}
\operatorname{Im} p & =\left\{\left(x+r_{+}(\xi), x+r_{-}(\xi)\right) \mid x \in \mathfrak{g}, \xi \in \mathfrak{g}^{*}\right\} \\
& =\left\{\left(x_{0}, x_{0}+y\right) \mid x_{0} \in \mathfrak{g}, y \in \mathfrak{f}\right\} \\
& \cong \mathfrak{g} \ltimes \mathfrak{f} .
\end{aligned}
$$

In the semidirect sum $\mathfrak{g} \ltimes \mathfrak{f}, \mathfrak{g}$ acts on $\mathfrak{f}$ via the adjoint action (recall that $\mathfrak{f}$ is an ideal of $\mathfrak{g})$. The resulting map $\mathcal{D}(\mathfrak{g}) \rightarrow \mathfrak{g} \ltimes \mathfrak{f}$, given by $x+\xi \mapsto\left(x+r_{+}(\xi),-\left(r_{+}-\right.\right.$ $\left.\left.r_{-}\right) \xi\right), x \in \mathfrak{g}, \xi \in \mathfrak{g}^{*}$ will still be denoted by $p$. This map was also constructed and discussed by Majid [8, 8.2.6].

Similarly, from (3.4) we see that the kernel of $p$ is

$$
\begin{aligned}
\operatorname{Ker} p & =\operatorname{Ker} p_{+} \cap \operatorname{Ker} p_{-} \\
& =\left(\mathrm{id}-r_{+}\right)\left(\operatorname{Ker}\left(r_{+}-r_{-}\right)\right) \\
& =\left(\mathrm{id}-r_{+}\right) \mathfrak{f}^{\perp} .
\end{aligned}
$$

Lemma 3.3 implies that $\operatorname{Ker} p$ is an abelian subalgebra of $\mathcal{D}(\mathfrak{g})$.

Equip the space $\mathfrak{f}^{\perp}$ with the structure of abelian Lie algebra. Then the map

$$
i=\left.\left(\mathrm{id}-r_{+}\right)\right|_{\mathfrak{f}^{\perp}}: \mathfrak{f}^{\perp} \rightarrow \mathcal{D}(\mathfrak{g})
$$

is an embedding of Lie algebras.

To summarize:

Proposition 3.4. The sequence

$$
0 \rightarrow \mathfrak{f}^{\perp} \stackrel{i}{\rightarrow} \mathcal{D}(\mathfrak{g}) \stackrel{p}{\rightarrow} \mathfrak{g} \ltimes \mathfrak{f} \rightarrow 0
$$

is exact.

Note that $\mathfrak{f}^{\perp}$, considered as a subspace of $\mathfrak{g}^{*}$, is not an abelian Lie algebra in general. For example if $\mathfrak{g}$ is a triangular Lie bialgebra, $\mathfrak{f}^{\perp}=\mathfrak{g}^{*}$ but $\mathfrak{g}^{*}$ is not always abelian.

\section{Description of the extension}

According to the previous section, $\mathcal{D}(\mathfrak{g})$ is an extension of $\mathfrak{g} \ltimes \mathfrak{f}$ by the abelian Lie algebra $\mathfrak{f}^{\perp}$. Thus, there is an induced representation of $\mathfrak{g} \ltimes \mathfrak{f}$ on $\mathfrak{f}^{\perp}$ and the extension is uniquely defined by an element of $H^{2}\left(\mathfrak{g} \ltimes \mathfrak{f}, \mathfrak{f}^{\perp}\right)$ (see, for instance, [1, XIV.5] or [4, VII.4]). To describe these structures, we need to fix a splitting $S: \mathfrak{g} \ltimes \mathfrak{f} \rightarrow \mathcal{D}(\mathfrak{g})$ of (3.12) in the category of vector spaces, i.e. $p \circ S=\mathrm{id}_{\mathfrak{g} \ltimes \mathfrak{f}}$, such that $\mathrm{S}(0)=0$. From (3.9) one sees that such splitting may be defined by a right inverse map $s: \mathfrak{f} \rightarrow \mathfrak{g}^{*}$ of $\left(r_{+}-r_{-}\right): \mathfrak{g}^{*} \rightarrow \mathfrak{f}\left(\right.$ i.e. $\left.\left(r_{+}-r_{-}\right) \circ s=\mathrm{id}_{\mathfrak{f}}\right)$. The desired splitting $S: \mathfrak{g} \ltimes \mathfrak{f} \rightarrow \mathcal{D}(\mathfrak{g})$ can then be defined by

$$
S(x, y)=x+r_{+} s(y)-s(y) \in \mathcal{D}(\mathfrak{g}), x \in \mathfrak{g}, y \in \mathfrak{f}
$$

(the elements of $\mathfrak{g} \ltimes \mathfrak{f}$ will be represented as ordered pairs).

Lemma 4.1. The maps id $-r_{ \pm}: \mathfrak{g}^{*} \rightarrow \mathcal{D}(\mathfrak{g})$ are $\mathfrak{g}$-module homomorphisms. That is, $\left(\mathrm{id}-r_{ \pm}\right)\left(\operatorname{ad}_{x}^{*} \xi\right)=\left[x,\left(\mathrm{id}-r_{ \pm}\right) \xi\right]$ for all $x \in \mathfrak{g}$ and all $\xi \in \mathfrak{g}^{*}$. 
Proof. In the $r_{+}$case we have

$$
\begin{aligned}
{\left[x,\left(\mathrm{id}-r_{+}\right) \xi\right] } & =-\left[x, r_{+} \xi\right]+\operatorname{ad}_{x}^{*} \xi-\operatorname{ad}_{\xi}^{*} x \\
& =-(\xi \otimes \mathrm{id})\left(\mathrm{id} \otimes \operatorname{ad}_{x}\right) r+\operatorname{ad}_{x}^{*} \xi-\operatorname{ad}_{\xi}^{*} x \\
& =\operatorname{ad}_{x}^{*} \xi+(\xi \otimes \mathrm{id})\left(\operatorname{ad}_{x} \otimes \mathrm{id}\right) r \\
& =\left(\mathrm{id}-r_{+}\right)\left(\operatorname{ad}_{x}^{*} \xi\right) .
\end{aligned}
$$

The argument for $r_{-}$is analogous.

Lemma 4.2. The action of $\mathfrak{g} \ltimes \mathfrak{f}$ on $\mathfrak{f}^{\perp}$ induced from the extension

$$
0 \rightarrow \mathfrak{f}^{\perp} \stackrel{i}{\rightarrow} \mathcal{D}(\mathfrak{g}) \stackrel{p}{\rightarrow} \mathfrak{g} \ltimes \mathfrak{f} \rightarrow 0
$$

is given by $(x, y) \cdot \eta=\operatorname{ad}_{x}^{*}(\eta)$ for $x \in \mathfrak{g}, y \in \mathfrak{f}$, and $\eta \in \mathfrak{f}^{\perp}$.

Proof. The action is given by $(x, y) \cdot \eta=i^{-1}([S(x, y), i(\eta)])$. Now Lemma 3.3 implies that $i\left(\mathfrak{f}^{\perp}\right)$ is in the center of Ker $p_{+}$and therefore

$$
\begin{aligned}
{[S(x, y), i(\eta)] } & =\left[x-\left(\mathrm{id}-r_{+}\right) s(y),\left(\mathrm{id}-r_{+}\right) \eta\right]=\left[x,\left(\mathrm{id}-r_{+}\right) \eta\right] \\
& =\left(\mathrm{id}-r_{+}\right)\left(\operatorname{ad}_{x}^{*}(\eta)\right) .
\end{aligned}
$$

by Lemma 4.1 .

Let us briefly recall the construction of an extension of a Lie algebra $\mathfrak{h}$ by an $\mathfrak{h}$-module $V$ associated to an element of $H^{2}(\mathfrak{h}, V)$. Let $\alpha \in Z^{2}(\mathfrak{h}, V)$ be a representative of the cohomology class $[\alpha] \in H^{2}(\mathfrak{h}, V)$. Then the extension of $\mathfrak{h}$ by $V$ associated to $\alpha$ is the vector space $\mathfrak{h} \oplus V$ equipped with the bracket

$$
\left[\left(h_{1}, v_{1}\right),\left(h_{2}, v_{2}\right)\right]=\left(\left[h_{1}, h_{2}\right], h_{1} \cdot v_{2}-h_{2} \cdot v_{1}+\alpha\left(h_{1}, h_{2}\right)\right) .
$$

We will denote such an extension by $\mathfrak{h} \ltimes_{\alpha} V$.

Returning to $\mathcal{D}(\mathfrak{g})$, notice that it follows easily from Lemma 4.1 that for any $x \in \mathfrak{g}$ and $y \in \mathfrak{f}$,

$$
\operatorname{ad}_{x}^{*}(s(y))-s([x, y]) \in \mathfrak{f}^{\perp} .
$$

Hence we may define a 2 -form, $\alpha:(\mathfrak{g} \ltimes \mathfrak{f}) \otimes(\mathfrak{g} \ltimes \mathfrak{f}) \rightarrow \mathfrak{f}^{\perp}$ by

$$
\begin{aligned}
\alpha\left(\left(x_{1}, y_{1}\right),\left(x_{2}, y_{2}\right)\right) & =-\operatorname{ad}_{x_{1}}^{*}\left(s\left(y_{2}\right)\right)+\operatorname{ad}_{x_{2}}^{*}\left(s\left(y_{1}\right)\right)-\operatorname{ad}_{y_{1}}^{*}\left(s\left(y_{2}\right)\right) \\
& +s\left(\left[x_{1}, y_{2}\right]-\left[x_{2}, y_{1}\right]+\left[y_{1}, y_{2}\right]\right) .
\end{aligned}
$$

Theorem 4.3. The form $\alpha$ is a 2-cocycle and

$$
\mathcal{D}(\mathfrak{g}) \cong(\mathfrak{g} \ltimes \mathfrak{f}) \ltimes_{\alpha} \mathfrak{f}^{\perp} .
$$

Proof. The cohomology class associated to the extension

$$
0 \rightarrow \mathfrak{f}^{\perp} \stackrel{i}{\rightarrow} \mathcal{D}(\mathfrak{g}) \stackrel{p}{\rightarrow} \mathfrak{g} \ltimes \mathfrak{f} \rightarrow 0
$$

is the class of the 2-cocycle

$$
i^{-1}\left(\left[S\left(x_{1}, y_{1}\right), S\left(x_{2}, y_{2}\right)\right]-S\left(\left[\left(x_{1}, y_{1}\right),\left(x_{2}, y_{2}\right)\right]\right)\right) .
$$


But

$$
\begin{aligned}
& {\left[S\left(x_{1}, y_{1}\right), S\left(x_{2}, y_{2}\right)\right]=\left[x_{1}-\left(\mathrm{id}-r_{+}\right) s\left(y_{1}\right), x_{2}-\left(\mathrm{id}-r_{+}\right) s\left(y_{2}\right)\right]} \\
& =\left[x_{1}, x_{2}\right]+\left(\mathrm{id}-r_{+}\right)\left(-\operatorname{ad}_{x_{1}}^{*}\left(s\left(y_{2}\right)\right)+\operatorname{ad}_{x_{2}}^{*}\left(s\left(y_{1}\right)\right)-\operatorname{ad}_{y_{1}}^{*}\left(s\left(y_{2}\right)\right)\right),
\end{aligned}
$$

and

$$
S\left(\left[\left(x_{1}, y_{1}\right),\left(x_{2}, y_{2}\right)\right]\right)=\left[x_{1}, x_{2}\right]-\left(\mathrm{id}-r_{+}\right)\left(\left[x_{1}, y_{2}\right]-\left[x_{2}, y_{1}\right]+\left[y_{1}, y_{2}\right]\right) .
$$

So

$$
\left[S\left(x_{1}, y_{1}\right), S\left(x_{2}, y_{2}\right)\right]-S\left(\left[\left(x_{1}, y_{1}\right),\left(x_{2}, y_{2}\right)\right]\right)=\left(\mathrm{id}-r_{+}\right)\left(\alpha\left(\left(x_{1}, y_{1}\right),\left(x_{2}, y_{2}\right)\right)\right)
$$

as required.

Remark 4.4. In the case when $\mathfrak{g}$ admits a nondegenerate invariant bilinear form, $\mathfrak{g}^{*}$ can be identified as a linear space with $\mathfrak{g}$ and $r_{ \pm}$can be thought of as linear endomorphisms of $\mathfrak{g}$. In Theorem $4.3 \mathfrak{f}^{\perp}$ may be replaced by the orthogonal complement to $\mathfrak{f}$ in $\mathfrak{g}$ and the coadjoint representation $\mathrm{ad}^{*}$ of $\mathfrak{g}$ with the adjoint one. The results of the following section can be reformulated in a similar way.

\section{The embeddings of $\mathfrak{g}$ and $\mathfrak{g}^{*}$ in $\mathcal{D}(\mathfrak{g})$}

It is clear that in the identification $(4.3), \mathfrak{g}$ is embedded in $\mathcal{D}(\mathfrak{g})$ by

$$
x \mapsto(x, 0) \ltimes_{\alpha} 0 \in(\mathfrak{g} \ltimes \mathfrak{f}) \ltimes_{\alpha} \mathfrak{f}^{\perp}, x \in \mathfrak{g}
$$

(see eqs. (3.11) and (4.1)).

From the definition of $s$ it follows that for any $\xi \in \mathfrak{g}^{*}$

$$
\xi-s\left(r_{+}-r_{-}\right) \xi \in \mathfrak{f}^{\perp} \text {. }
$$

This easily gives

$$
\xi=S\left(r_{+}(\xi),-\left(r_{+}-r_{-}\right) \xi\right)+i\left(\xi-s\left(r_{+}-r_{-}\right) \xi\right), \forall \xi \in \mathfrak{g}^{*} .
$$

Thus the embedding of $\mathfrak{g}^{*}$ in $(\mathfrak{g} \ltimes \mathfrak{f}) \ltimes_{\alpha} \mathfrak{f}^{\perp} \cong \mathcal{D}(\mathfrak{g})$ is given by

$$
\xi \mapsto\left(r_{+}(\xi),-\left(r_{+}-r_{-}\right) \xi\right) \ltimes_{\alpha}\left(\xi-s\left(r_{+}-r_{-}\right) \xi\right) .
$$

In this section we describe in an invariant way the image of this embedding.

It will be more convenient to work with the algebra

$$
\mathfrak{a}:=\left\{\left(x_{1}, x_{2}\right) \mid x_{i} \in \mathfrak{g}, x_{2}-x_{1} \in \mathfrak{f}\right\} \subset \mathfrak{g} \oplus \mathfrak{g}
$$

isomorphic to $\mathfrak{g} \ltimes \mathfrak{f}$. The corresponding isomorphism as shown in (3.9) is $\left(x_{1}, x_{2}\right) \mapsto\left(x_{1}, x_{2}-x_{1}\right)$. In terms of $\mathfrak{a}$ the identification (4.3) is

$$
\mathcal{D}(\mathfrak{g}) \cong \mathfrak{a} \ltimes_{\alpha} \mathfrak{f}^{\perp}
$$

and the corresponding action of $\mathfrak{a}$ on $\mathfrak{f}^{\perp}$ is

$$
\left(x_{1}, x_{2}\right) \cdot \eta=\operatorname{ad}_{x_{1}}^{*}(\eta) \in \mathfrak{f}^{\perp},\left(x_{1}, x_{2}\right) \in \mathfrak{a}, \eta \in \mathfrak{f}^{\perp} .
$$

It is also straightforward to write $\alpha$ as a 2 -cocycle for $\mathfrak{a}$ with values in $\mathfrak{f}^{\perp}$. From (5.2) it follows that $\mathfrak{g}^{*}$ is embedded in $\mathfrak{a} \ltimes_{\alpha} \mathfrak{f}^{\perp} \cong \mathcal{D}(\mathfrak{g})$ by

$$
\xi \mapsto\left(r_{+}(\xi), r_{-}(\xi)\right) \ltimes_{\alpha}\left(\xi-s\left(r_{+}-r_{-}\right) \xi\right), \xi \in \mathfrak{g}^{*} .
$$


We first state a general classification of subalgebras of extensions of the form $\mathfrak{h} \ltimes_{\alpha} V$ where $V$ is an $\mathfrak{h}$-module and $\alpha \in Z^{2}(\mathfrak{h}, V)$.

Lemma 5.1. Let $\mathfrak{b} \subset \mathfrak{h}$, let $W$ be a $\mathfrak{b}$-submodule of $V$ and let $\sigma$ be the projection $V \rightarrow V / W$. Let $\gamma: \mathfrak{b} \rightarrow V / W$ be a 1-cochain whose coboundary is $-\left.\sigma \circ \alpha\right|_{\mathfrak{b}}$. Define

$$
\mathfrak{p}(\gamma, \sigma)=\{(x, v) \mid x \in \mathfrak{b}, \gamma(x)=\sigma(v)\} .
$$

Then $\mathfrak{p}(\gamma, \sigma)$ is a Lie subalgebra of $\mathfrak{h} \ltimes_{\alpha} V$.

Proof. First suppose that $W=0$. Then an arbitrary element of $\mathfrak{p}(\gamma, \sigma)$ is of the form $(x, \gamma(x))$ and

$$
\left[\left(x_{1}, \gamma\left(x_{1}\right)\right),\left(x_{2}, \gamma\left(x_{2}\right)\right)\right]=\left(\left[x_{1}, x_{2}\right], \alpha\left(x_{1}, x_{2}\right)+x_{1} \cdot \gamma\left(x_{2}\right)-x_{2} \cdot \gamma\left(x_{1}\right)\right) .
$$

Now $d \gamma=-\alpha$ implies that $x_{1} \cdot \gamma\left(x_{2}\right)-x_{2} \cdot \gamma\left(x_{1}\right)-\gamma\left(x_{1}, x_{2}\right)=-\alpha\left(x_{1}, x_{2}\right)$ so that

$$
\left[\left(x_{1}, \gamma\left(x_{1}\right)\right),\left(x_{2}, \gamma\left(x_{2}\right)\right)\right]=\left(\left[x_{1}, x_{2}\right], \gamma\left(\left[x_{1}, x_{2}\right]\right)\right) .
$$

Thus $\mathfrak{p}(\gamma, \sigma)$ is a Lie subalgebra of $\mathfrak{h} \ltimes_{\alpha} V$. In the more general situation, we notice that $\mathfrak{p}(\gamma, \sigma)$ is the inverse image of $\mathfrak{p}\left(\gamma,\left.\mathrm{id}\right|_{V / W}\right)$ under the natural projection $\mathfrak{b} \ltimes_{\alpha} V \rightarrow \mathfrak{b} \ltimes_{\bar{\alpha}} V / W$, where $\bar{\alpha}=\left.\sigma \circ \alpha\right|_{\mathfrak{b}}$.

Let $p: \mathfrak{h} \ltimes_{\alpha} V \rightarrow \mathfrak{h}$ and $q: \mathfrak{h} \ltimes_{\alpha} V \rightarrow V$ be the projections $p(h, v)=h$ and $q(h, v)=v$. (Recall from the previous section that the map (3.8) is a special case of the first projection.)

Theorem 5.2. Let $\mathfrak{k}$ be a Lie subalgebra of $\mathfrak{h} \ltimes_{\alpha} V$; let $\mathfrak{b}=p(\mathfrak{k}), W=\mathfrak{k} \cap V$ and let $\sigma: V \rightarrow V / W$ be the natural projection. Then $\mathfrak{k}=\mathfrak{p}(\gamma, \sigma)$ where $\gamma: \mathfrak{b} \rightarrow V / W$ is given by $\gamma(x)=q\left(p^{-1}(x)\right)+W$.

Proof. If $x \in \mathfrak{b}$ and $(x, v)$ and $\left(x, v^{\prime}\right)$ belong to $\mathfrak{k}$, then $v-v^{\prime} \in W$, so $q\left(p^{-1}(x)\right)$ is an element of $V / W$. Thus $\gamma$ is well-defined and $\mathfrak{k}=\{(x, v) \mid x \in \mathfrak{b}, \sigma(v)=\gamma(x)\}$. It remains to check that $d \gamma=-\left.\sigma \circ \alpha\right|_{\mathfrak{b}}$. But since

$$
\left[\left(x_{1}, v_{1}\right),\left(x_{2}, v_{2}\right)\right]=\left(\left[x_{1}, x_{2}\right], \alpha\left(x_{1}, x_{2}\right)+x_{1} \cdot v_{2}-x_{2} \cdot v_{1}\right)
$$

we must have that $\gamma\left(\left[x_{1}, x_{2}\right]\right)=\sigma\left(\alpha\left(x_{1}, x_{2}\right)+x_{1} \cdot v_{2}-x_{2} \cdot v_{1}\right)$. Hence

$$
\sigma \circ \alpha\left(x_{1}, x_{2}\right)=\gamma\left(\left[x_{1}, x_{2}\right]\right)-x_{1} \cdot \gamma\left(v_{2}\right)+x_{2} \cdot \gamma\left(v_{1}\right)
$$

as required.

We now identify the image of $\mathfrak{g}^{*}$ inside $\mathfrak{a} \ltimes_{\alpha} \mathfrak{f}^{\perp}$. It follows from (5.2) that $W=\operatorname{Ker} r_{+} \cap \operatorname{Ker} r_{-}$. The projection $\mathfrak{b}=p\left(\mathfrak{g}^{*}\right)$ can be given the following conceptual description.

Define $\mathfrak{g}_{ \pm}=\operatorname{Im} r_{ \pm}$and $\mathfrak{n}_{ \pm}=r_{ \pm}\left(\operatorname{Ker} r_{\mp}\right)$. Recall from $[9,10]$ that $\mathfrak{n}_{ \pm}$are ideals of $\mathfrak{g}_{ \pm}$. The map $\theta: \mathfrak{g}_{+} / \mathfrak{n}_{+} \rightarrow \mathfrak{g}_{-} / \mathfrak{n}_{-}$given by

$$
\theta\left(r_{+}(\xi)+\mathfrak{n}_{+}\right)=r_{-}(\xi)+\mathfrak{n}_{-}, \xi \in \mathfrak{g}^{*}
$$

is well defined and is an isomorphism of Lie algebras. It is called the Cayley transform of the $r$-matrix $r$. The projections from $\mathfrak{g}_{ \pm}$to $\mathfrak{g}_{ \pm} / \mathfrak{n}_{ \pm}$will be denoted by $\pi_{ \pm}$. 
Let $\mathfrak{b}$ be the subalgebra of $\mathfrak{a} \cap\left(\mathfrak{g}_{+} \oplus \mathfrak{g}_{-}\right) \subset \mathfrak{g} \oplus \mathfrak{g}$ consisting of the elements $\left(x_{+}, x_{-}\right) \in \mathfrak{g}_{+} \oplus \mathfrak{g}_{-}$such that

$$
\theta \circ \pi_{+}\left(x_{+}\right)=\pi_{-}\left(x_{-}\right) .
$$

The following lemma is a direct consequence from the definition of $\theta$ (cf. [10]).

Lemma 5.3. The image of $\mathfrak{g}^{*} \subset \mathcal{D}(\mathfrak{g})$ under the projection $p: \mathcal{D}(\mathfrak{g}) \rightarrow \mathfrak{a}$ coincides with $\mathfrak{b} \subset \mathfrak{a}:$

$$
p\left(\mathfrak{g}^{*}\right)=\mathfrak{b}
$$

The projection $\left.p\right|_{\mathfrak{g}^{*}}: \mathfrak{g}^{*} \rightarrow \mathfrak{a}$ factors through to an isomorphism $\bar{p}: \mathfrak{g}^{*} / W \rightarrow \mathfrak{b}$ given by

$$
\bar{p}(\xi+W)=\left(r_{+}(\xi), r_{-}(\xi)\right) .
$$

Hence the 1-cochain $\gamma: \mathfrak{b} \rightarrow \overline{\mathfrak{f}}^{\perp}$ of Theorem 5.2 in this situation will be given by

$$
\begin{aligned}
\gamma\left(x_{1}, x_{2}\right) & =\bar{p}^{-1}\left(x_{1}, x_{2}\right)-\sigma s\left(r_{+}-r_{-}\right) \bar{p}^{-1}\left(x_{1}, x_{2}\right) \\
& =\bar{p}^{-1}\left(x_{1}, x_{2}\right)-\sigma s\left(x_{1}-x_{2}\right) .
\end{aligned}
$$

This description can be summarized as follows.

Theorem 5.4. The image of $\mathfrak{g}^{*}$ inside $\mathcal{D}(\mathfrak{g})$ under the isomorphism $\mathcal{D}(\mathfrak{g}) \cong$ $\mathfrak{a} \ltimes_{\alpha} \mathfrak{f}^{\perp}$ coincides with the subalgebra $\mathfrak{p}(\gamma, \sigma)$ where $\mathfrak{b}$ is the algebra described in Lemma 5.3, $\sigma$ is the projection $V \rightarrow V / W$ for $W=\operatorname{Ker} r_{+} \cap \operatorname{Ker} r_{-}$and $\gamma: \mathfrak{b} \rightarrow \mathfrak{f}^{\perp} / W$ is as described in (5.8).

Remark 5.5. To describe the Manin triple $\left(\mathcal{D}(\mathfrak{g}), \mathfrak{g}, \mathfrak{g}^{*}\right)$ completely, we are left with expressing the canonical bilinear form $(2.4)$ on $\mathcal{D}(\mathfrak{g})$ in terms of the identification $\mathcal{D}(\mathfrak{g}) \cong \mathfrak{a} \ltimes_{\alpha} \mathfrak{f}^{\perp}$. This is needed for example for the description of the Poisson homogeneous spaces for the Poisson-Lie groups with tangent Lie bialgebra $\mathfrak{g}$ as explained in the introduction. For any

$$
d=\left(x_{1}, x_{2}\right) \ltimes_{\alpha} \eta \in \mathfrak{a} \ltimes_{\alpha} \mathfrak{f}^{\perp}
$$

set

$$
\begin{aligned}
& \xi(d):=\eta+s\left(r_{+}-r_{-}\right)\left(x_{1}-x_{2}\right), \\
& x(d):=x_{1}-r_{+}(x(d)) .
\end{aligned}
$$

Using equations (5.1) and (5.6), it is easy to show that the composition of the isomorphisms $\mathfrak{a} \ltimes_{\alpha} \mathfrak{f}^{\perp} \cong \mathcal{D}(\mathfrak{g}) \cong \mathfrak{g}+\mathfrak{g}^{*}$ is given by $d \mapsto(x(d), \xi(d))$. This implies that the bilinear form (2.4) on $\mathfrak{a} \ltimes_{\alpha} \mathfrak{f}^{\perp} \cong \mathcal{D}(\mathfrak{g})$ is given by

$$
\left\langle\left\langle d_{1}, d_{2}\right\rangle\right\rangle:=\left\langle\xi\left(d_{1}\right), x\left(d_{2}\right)\right\rangle+\left\langle\xi\left(d_{2}\right), x\left(d_{1}\right)\right\rangle, d_{i} \in \mathfrak{a} \ltimes_{\alpha} \mathfrak{f}^{\perp} .
$$




\section{Applications to Poisson-Lie groups}

Let $G$ be a finite dimensional connected, simply connected quasitriangular Poisson-Lie group with Lie bialgebra $\mathfrak{g}$. (The ground field $k$ is assumed to be $\mathbb{R}$ or C.) As an application of Theorems 4.3 and 5.4, we explicitly construct the double and dual Poisson-Lie groups $\mathcal{D}(G), G^{*}$ of $G$. (Recall from the introduction that they are the simply connected Poisson-Lie groups with tangent Lie bialgebras $\mathcal{D}(\mathfrak{g})$ and $\mathfrak{g}^{*}$, respectively.) We also describe the image of $G^{*}$ under the induced map $j^{*}: G^{*} \rightarrow \mathcal{D}(G)$ from the embedding of $\mathfrak{g}^{*}$ into $\mathcal{D}(\mathfrak{g})$.

We start with some general remarks on group extensions. Assume that $H$ is a Lie group and $V$ is an $H$-module. A normalized analytic 2-cocycle $\mathcal{A} \in Z^{2}(H, V)$ gives rise to the extension $H \ltimes_{\mathcal{A}} V$ which as a manifold is $H \times V$ and has multiplication law

$$
\left(h_{1}, v_{1}\right)\left(h_{2}, v_{2}\right)=\left(h_{1} h_{2}, v_{1}+h_{1} \cdot v_{2}+\mathcal{A}\left(h_{1}, h_{2}\right)\right) .
$$

If $\mathfrak{h}=\operatorname{Lie}(H)$ and the derivative of $\mathcal{A}$ is $\alpha \in Z^{2}(\mathfrak{h}, V)$, then $\operatorname{Lie}\left(H \ltimes_{\mathcal{A}} V\right) \cong$ $\mathfrak{h} \ltimes_{\alpha} V$. When $H$ is simply connected any such Lie algebra cocycle $\alpha$ can be lifted (uniquely) to an analytic Lie group cocycle $\mathcal{A}[5]$.

Abstract subgroups of $H \ltimes_{\mathcal{A}} V$ can be described in a fashion analogous to Theorem 5.2. Let $B$ be a subgroup of $H$ and let $U$ be a subgroup of $V$ that is $B$-invariant. Denote by $\tau$ the projection $V \rightarrow V / U$. A 1-cochain $\Gamma: B \rightarrow V / U$ with coboundary $-\left.\tau \circ \mathcal{A}\right|_{B}$ gives rise to the subgroup

$$
P(\Gamma, \tau)=\{(b, v) \mid b \in B, \Gamma(b)=\tau(v)\}
$$

of $H \ltimes_{\mathcal{A}} V$. Analogously to Theorem 5.2, all abstract subgroups of $H \ltimes_{\mathcal{A}} V$ are obtained in this way. If $B$ is a Lie subgroup, $U$ is closed in $V$ and $\Gamma$ is analytic, then $P(\Gamma, \tau)$ is a Lie subgroup. Let $\mathfrak{b}$ be the Lie algebra of $B$, let $W$ be the connected component of $U$ containing 0 (which is of course a subspace of $V$ ) and let $\sigma$ be the projection $V \rightarrow V / W$. The derivative of $\Gamma$ is a map $\gamma: \mathfrak{b} \rightarrow V / W$ with coboundary $-\left.\sigma \circ \alpha\right|_{\mathfrak{b}}$. In the notation of Lemma $5.1, \operatorname{Lie}(P(\Gamma, \tau)) \cong \mathfrak{p}(\gamma, \sigma)$.

The converse is a little more subtle. Let $\mathfrak{b}$ be a Lie subalgebra of $\mathfrak{h}$ and let $B$ be the connected subgroup of $H$ corresponding to it. Fix a 1-cochain $\gamma$ of $\mathfrak{b}$ with values in $V / W$ and coboundary $-\left.\sigma \circ \alpha\right|_{\mathfrak{b}}$ and let $\mathfrak{p}(\gamma, \sigma)$ be the corresponding subalgebra of $\mathfrak{h} \ltimes_{\alpha} V$. Denote by $\eta: \widetilde{B} \rightarrow B$ the universal cover of $B$, and let $\mathcal{A}_{B}$ be the induced 2-cocycle on $\widetilde{B}$.

Lemma 6.1. The cochain $\gamma$ of $\mathfrak{b}$ can be integrated to a unique 1-cochain $\widetilde{\Gamma}$ on $\widetilde{B}$ with values in $V / W$ and coboundary $-\sigma \circ \mathcal{A}_{B}$. The subgroup $P(\tilde{\Gamma}, \sigma)$ of $\widetilde{B} \ltimes \mathcal{A}_{B} V$ is then a simply connected Lie group with Lie algebra $\mathfrak{p}(\gamma, \sigma)$. The embedding $\mathfrak{p}(\gamma, \sigma) \hookrightarrow \mathfrak{h} \ltimes_{\alpha} V$ yields a map $j^{*}: P(\tilde{\Gamma}, \sigma) \rightarrow H \ltimes_{\mathcal{A}} V$ given by $j^{*}\left(\widetilde{b} \ltimes_{\mathcal{A}_{B}} v\right)=\eta(\widetilde{b}) \ltimes_{\mathcal{A}} v$. Its image is the connected subgroup of $H \ltimes_{\mathcal{A}} V$ with Lie algebra $\mathfrak{p}(\gamma, \sigma)$ and is of the form $P(\Gamma, \tau)$ where $\tau: V \rightarrow V / \sigma^{-1}(\Gamma($ Ker $\eta))$.

Proof. Denote by $K$ the connected subgroup of $\widetilde{B} \ltimes \mathcal{A}_{B} V$ with Lie algebra $\mathfrak{p}(\gamma, \sigma)$. Clearly $K \supset W$ and the projection on the first component $p: K \rightarrow \widetilde{B}$ factors 
through to a projection $p_{W}: K / W \rightarrow \widetilde{B}$. The induced map on Lie algebras is an isomorphism. Since $\widetilde{B}$ is simply connected, $p_{W}$ is also an isomorphism and $K \cap V=W$. Hence $K$ is of the type $P(\tilde{\Gamma}, \sigma)$ for a suitable map $\widetilde{\Gamma}: \widetilde{B} \rightarrow V / W$. Now $\widetilde{\Gamma}$ must be an analytic cochain on $\widetilde{B}$ with coboundary $-\sigma \circ \mathcal{A}_{B}$ because $K$ is a Lie subgroup of $\widetilde{B} \ltimes_{\mathcal{A}_{B}} V$. The uniqueness of a cocycle $\widetilde{\Gamma}$ with the prescribed properties follows from the uniqueness of a connected Lie subgroup with given tangent Lie bialgebra.

The fact that $P(\tilde{\Gamma}, \sigma)$ is simply connected trivially follows from the simply connectedness of $\operatorname{Ker} p_{W}=W$ and $\operatorname{Im} p_{W}=\widetilde{B}$.

Denote by $F$ the connected subgroup of $G$ with $\operatorname{Lie}(F)=\mathfrak{f}$ and by $G \ltimes F$ the semidirect product where $G$ acts on $F$ by the adjoint action. Since $\mathfrak{f}$ is an ideal, $F$ is closed, normal and simply connected [13, 3.18]. Hence $G \ltimes F$ is also simply connected. As remarked above the 2-cocycle $\alpha$ of $\mathfrak{g} \ltimes \mathfrak{f}$ (see (4.2)) can be integrated to a 2-cocycle $\mathcal{A}(.,$.$) for the action of G \ltimes F$ on $\mathfrak{f}^{\perp}$ given by

$$
(g, f) \cdot(\eta):=\operatorname{Ad}_{g}^{*}(\eta)
$$

The derivative of this action is of course the action of $\mathfrak{g} \ltimes \mathfrak{f}$ on $\mathfrak{f}^{\perp}$ from Lemma 4.2 .

Consider the group

$$
D=(G \ltimes F) \ltimes_{\mathcal{A}} \mathfrak{f}^{\perp} .
$$

Since

$$
\operatorname{Lie}(D) \cong(\mathfrak{g} \ltimes \mathfrak{f}) \ltimes_{\alpha} \mathfrak{f}^{\perp}
$$

is isomorphic to the underlying Lie algebra of the double Lie bialgebra $\mathcal{D}(\mathfrak{g}), D$ can be equipped with the Sklyanin Poisson structure (see, for instance, [2]). The resulting Poisson-Lie group is the double of $G$. As in the Lie algebra case, we can equivalently use the group

$$
A=\left\{\left(g_{1}, g_{2}\right) \in G \times G \mid g_{1}^{-1} g_{2} \in F\right\} \cong G \ltimes F
$$

and write $\mathcal{A}$ as a 2-cocycle for the action of $A$ on $\mathfrak{f}^{\perp}$ induced from (6.1) (cf. also (5.5)). Then

$$
D \cong A \ltimes_{\mathcal{A}} \mathfrak{f}^{\perp}
$$

Denote by $G_{ \pm}$the connected subgroups of $G$ with tangent Lie algebras $\mathfrak{g}_{ \pm}$. Let $B$ be the connected subgroup of $G_{+} \times G_{-}$with $\operatorname{Lie}(B)=\mathfrak{b}$. If the isomorphism $\theta: \mathfrak{g}_{+} / \mathfrak{n}_{+} \rightarrow \mathfrak{g}_{-} / \mathfrak{n}_{-}$can be integrated to a group isomorphism $\Theta: G_{+} / N_{+} \rightarrow$ $G_{-} / N_{-}$for some closed (not necessarily connected) subgroups $N_{ \pm}$of $G$ with $\operatorname{Lie}\left(N_{ \pm}\right)=\mathfrak{n}_{ \pm}$(taken the minimal possible), then $B$ is explicitly given by

$$
B=\left\{\left(g_{1}, g_{2}\right) \in G_{+} \times G_{-} \mid \Theta \circ \Pi_{+}\left(g_{1}\right)=\Pi_{-}\left(g_{2}\right)\right\}
$$

where $\Pi_{ \pm}$denote the homomorphisms $G_{ \pm} \rightarrow G_{ \pm} / N_{ \pm}$. As above $\eta: \widetilde{B} \rightarrow B$ will denote the simply connected cover of $B$.

Combining Theorem 5.4 and Lemma 6.1 we obtain: 
Theorem 6.2. Let $G$ be a connected, simply connected quasitriangular PoissonLie group. Let $F$ be the connected normal Lie subgroup with Lie algebra $\mathfrak{f}$. Then the double Poisson-Lie group of $G$ is isomorphic, as a Lie group, to $(G \ltimes$ $F) \ltimes_{\mathcal{A}} \mathfrak{f}^{\perp} \cong A \ltimes_{\mathcal{A}} \mathfrak{f}^{\perp}$ where $\mathcal{A}$ is a normalized analytic 2-cocycle on $G \ltimes F$ with derivative $\alpha$. The dual Poisson-Lie group $G^{*}$ is of the form $P(\tilde{\Gamma}, \sigma)$ where $W=\operatorname{Ker} r_{+} \cap \operatorname{Ker} r_{-}, \sigma: V \rightarrow V / W$ and $\widetilde{\Gamma}: \widetilde{B} \rightarrow V / W$ is the unique analytic 1-cochain on $\widetilde{B}$ with coboundary $-\sigma \circ \mathcal{A}_{\vec{B}}$ and derivative $\gamma$. The image of $G^{*}$ in $\mathcal{D}(G)$ is of the form $P(\Gamma, \tau)$ where $U=\sigma^{-1}(\widetilde{\Gamma}($ Ker $\eta))$ is a Lie subgroup of $V$ with connected component $W, \tau: V \rightarrow V / U$ and $\Gamma: B \rightarrow V / U$ is a 1-cochain on $B$ induced from $\widetilde{\Gamma}$.

\section{Special cases}

In this section we treat some cases of the results from the previous ones and compare them to the available results in the literature.

7.1. Factorizable Lie bialgebras. Recall that a quasitriangular Lie bialgebra $(\mathfrak{g}, r)$ is called factorizable if $\Omega=r+r_{21} \in S^{2}(\mathfrak{g})$ is nondegenerate, or in other words if $\mathfrak{f}=\mathfrak{g}$. In this case $\mathfrak{f}^{\perp}=0$ and $p$ defines an isomorphism

$$
\mathcal{D}(\mathfrak{g}) \cong \mathfrak{a}=\mathfrak{g} \oplus \mathfrak{g} .
$$

The results from Section 5 reduce to the fact that in the above identification, $\mathfrak{g}^{*} \subset \mathcal{D}(\mathfrak{g})$ is isomorphic to the subalgebra $\mathfrak{b} \subset \mathfrak{g}_{+} \oplus \mathfrak{g}_{-}$, defined in (5.7). This recovers the following result of Reshetikhin and Semenov-Tian-Shansky [9, 10].

Corollary 7.1. The double of a factorizable Lie bialgebra $\mathfrak{g}$ is isomorphic, as a Lie algebra, to $\mathfrak{g} \oplus \mathfrak{g}$. Under this isomorphism, the dual $\mathfrak{g}^{*}$ coincides with the subalgebra $\mathfrak{b}$ of $\mathfrak{g} \oplus \mathfrak{g}$ defined in (5.7) and $\mathfrak{g}$ with its diagonal subalgebra.

In particular Corollary 7.1 and Section 6 imply that the double of any simply connected factorizable Poisson-Lie group is isomorphic to $G \times G$ as a Lie group. The image of the dual Poisson-Lie group of $G$ inside $\mathcal{D}(G)$ coincides with the group $B$ constructed in Section 6, see (6.2).

7.2. Triangular Lie bialgebras. Let $(\mathfrak{g}, r)$ be a triangular Lie bialgebra, that is $r_{21}=-r$. This means that $\Omega=0$ and consequently $\mathfrak{f}=0, \mathfrak{f}^{\perp}=\mathfrak{g}^{*}$. Hence the map $s$ is trivial and the exact sequence (3.12) naturally splits $(\alpha=0)$.

Corollary 7.2. The double $\mathcal{D}(\mathfrak{g})$ of a triangular Lie bialgebra $\mathfrak{g}$ is isomorphic as a Lie algebra to the semidirect sum of $\mathfrak{g}$ and the $\mathfrak{g}$-module $\mathfrak{g}^{*}$

$$
\mathcal{D}(\mathfrak{g}) \cong \mathfrak{g} \ltimes \mathfrak{g}^{*}
$$

where $\mathfrak{g}$ acts on $\mathfrak{g}^{*}$ via the coadjoint action.

Next we specialize the results from Section 5 to the triangular case. The Lie algebras coincides with $\mathfrak{g}_{+}=\mathfrak{g}_{-}$. The space $W$ is Ker $r_{+}$and thus $\mathfrak{g}^{*} / W \cong \mathfrak{g}_{+}^{*}$. 
The map $r_{+}: \mathfrak{g}^{*} \rightarrow \mathfrak{g}_{+}$factors through to an isomorphism $\bar{r}_{+}: \mathfrak{g}^{*} / W \rightarrow \mathfrak{g}_{+}$. Its inverse

$$
\gamma:=\bar{r}_{+}^{-1}: \mathfrak{g}_{+} \rightarrow \mathfrak{g}^{*} / \text { Ker } r_{+} \cong \mathfrak{g}_{+}^{*}
$$

is a 1-cocycle for the coadjoint representation of $\mathfrak{g}_{+}$.

Corollary 7.3. For a triangular Lie bialgebra $\mathfrak{g}$, the image of the embedding $\mathfrak{g}^{*} \subset \mathcal{D}(\mathfrak{g}) \cong \mathfrak{g} \ltimes \mathfrak{g}^{*}$ coincides with $\mathfrak{p}(\gamma, \sigma) \subset \mathfrak{g}_{+} \ltimes \mathfrak{g}^{*}$ where $\sigma: \mathfrak{g}^{*} \rightarrow \mathfrak{g}^{*} / \operatorname{Ker} r_{+}$ and $\gamma$ is the 1-cocycle (7.2) for the coadjoint representation of $\mathfrak{g}_{+}$.

Notice that the image of $\mathfrak{g}^{*}$ in $\mathcal{D}(\mathfrak{g})$ can be more simply described as

$$
\left\{\left(r_{+}(\xi), \xi\right) \mid \xi \in \mathfrak{g}^{*}\right\} \subset \mathfrak{g} \ltimes \mathfrak{g}^{*} .
$$

However, this description, although explicit, is less useful because it cannot be lifted directly to the level of Poisson-Lie groups.

Let $G$ be a simply connected triangular Poisson-Lie group and $\mathfrak{g}=\operatorname{Lie}(G)$. Then the double Poisson-Lie group of $G$ is isomorphic, as a Lie group, to $G \ltimes \mathfrak{g}^{*}$. The dual Poisson-Lie group $G^{*}$ of $G$ is isomorphic to $P(\widetilde{\Gamma}, \sigma)$ where $\eta: \widetilde{G}_{+} \rightarrow G_{+}$ is the simply connected cover of $G_{+}, \widetilde{\Gamma}: \widetilde{G}_{+} \rightarrow \mathfrak{g}^{*} / \operatorname{Ker} r_{+}$is the unique 1-cocycle on $\widetilde{G}_{+}$with derivative $\gamma$ and $\sigma: \mathfrak{g}^{*} \rightarrow \mathfrak{g}^{*} / \operatorname{Ker} r_{+}$. The image of $G^{*}$ in $G \ltimes \mathfrak{g}^{*}$ will be of the form $P(\Gamma, \tau)$ where $U=\sigma^{-1}(\widetilde{\Gamma}($ Ker $\eta)), \Gamma: G_{+} \rightarrow \mathfrak{g}^{*} / U$ is the 1-cochain on $G_{+}$induced from $\widetilde{\Gamma}$, and $\tau: \mathfrak{g}^{*} \rightarrow \mathfrak{g}^{*} / U$ is the projection. These results can be easily restated in terms of $T^{*} G \cong G \ltimes \mathfrak{g}^{*}$.

Notice that if $\gamma$ is a coboundary (i.e., of the form $\gamma(x)=a d_{x}^{*}(\xi)$ for some $\left.\xi \in \mathfrak{g}_{+}^{*}\right)$ then $\widetilde{\Gamma}: \widetilde{G}_{+} \rightarrow \mathfrak{g}_{+}^{*} \cong \mathfrak{g}^{*} /$ Kerr $r_{+}$is just the map $\widetilde{\Gamma}(g)=\left(\operatorname{Ad}_{g}^{*}-\mathrm{id}\right) \xi$. Since Ker $\eta$ acts trivially on $\mathfrak{g}^{*}, U=\operatorname{Ker} r_{+}$and $\Gamma: \widetilde{G}_{+} \rightarrow \mathfrak{g}_{+}^{*} \cong \mathfrak{g}^{*} / \operatorname{Ker} r_{+}$is given by the same formula as $\widetilde{\Gamma}$.

Given the (nondegenerate) 1 -cocycle $\gamma$ of $\mathfrak{g}_{+}$with values in $\mathfrak{g}_{+}^{*}$, one can define a (nondegenerate) 2-cocycle $c(.,$.$) of \mathfrak{g}_{+}$with values in the ground field $k$ defined by

$$
c\left(x_{1}, x_{2}\right)=\left\langle\gamma\left(x_{1}\right), x_{2}\right\rangle, x_{i} \in \mathfrak{g}_{+} .
$$

This makes the pair $\left(\mathfrak{g}_{+}, c\right)$ a quasi-Frobenius Lie algebra, see [12]. When $\gamma$ is nondegenerate, so is $c$ and the pair $\left(\mathfrak{g}_{+}, c\right)$ becomes a Frobenius Lie algebra.

In the special case of a triangular bialgebra structure on a real compact form $\mathfrak{g}$ of a complex simple Lie algebra $\mathfrak{g}_{\mathbb{C}}$, Corollaries 7.2 and 7.3 were obtained by Levendorskii and Soibelman [7]. Although in this case additional simplifications occur $(r \in \mathfrak{h} \wedge \mathfrak{h}$ for a Cartan subalgebra $\mathfrak{h}$ of $\mathfrak{g})$, their proof can be extended to the general case of triangular bialgebras. As the one by Reshetikhin and Semenov-Tian-Shansky [9], it relies on a direct construction of a Manin triple.

Sketch of a second proof of Corollary 7.2. Let $\mathfrak{d}$ denote the semidirect sum $\mathfrak{g} \ltimes$ $\mathfrak{g}^{*}$, defined in Corollary 7.2. Equip it with the following natural invariant bilinear form

$$
\left\langle\left(x_{1}, \xi_{1}\right),\left(x_{2}, \xi_{2}\right)\right\rangle=\left\langle\xi_{1}, x_{2}\right\rangle+\left\langle\xi_{2}, x_{1}\right\rangle, \forall x_{i} \in \mathfrak{g}, \xi_{i} \in \mathfrak{g}^{*}
$$


It is clear that this form is nondegenerate and ad invariant. Define the linear maps:

$$
\begin{aligned}
& j: \mathfrak{g} \rightarrow \mathfrak{d}, j(x)=(x, 0), \\
& j^{*}: \mathfrak{g}^{*} \rightarrow \mathfrak{d}, j^{*}(\xi)=\left(r_{+}(\xi), \xi\right) .
\end{aligned}
$$

By a direct computation one checks that $j$ and $j^{*}$ are homomorphisms of Lie algebras and the images $j(\mathfrak{g}) \cong \mathfrak{g}, j^{*}\left(\mathfrak{g}^{*}\right) \cong \mathfrak{g}^{*}$ are Lagrangian subalgebras of $\mathfrak{d}$. Thus $\left(\mathfrak{d}, j(\mathfrak{g}), j^{*}\left(\mathfrak{g}^{*}\right)\right)$ is a Manin triple and $\mathcal{D}(\mathfrak{g}) \cong \mathfrak{d}$ as a Lie algebra.

If the Lie algebra $\mathfrak{g}$ admits an invariant bilinear form, $\mathfrak{g}^{*}$ can be identified, as a vector space, with $\mathfrak{g}$ and the coadjoint action substituted with the adjoint one. In this case Corollary 7.2 simply means that

$$
\mathcal{D}(\mathfrak{g}) \cong \mathfrak{g}[t] /\left\langle\left(t^{2}\right)\right\rangle
$$

where $t$ is a free variable. In addition $W=\operatorname{Ker} r_{+}$can be identified with $\mathfrak{g}_{+}^{\perp}$ and $\sigma$ thought of as projection $\mathfrak{g} \rightarrow \mathfrak{g} / \mathfrak{g}_{+}^{\perp}$. Then $\gamma$ is a 1 -cocycle of $\mathfrak{g}_{+}$with values in $\mathfrak{g} / \mathfrak{g}_{+}^{\perp}$ made a $\mathfrak{g}_{+}$module using the adjoint action. (Everywhere the orthogonal complement is taken with respect to the nondegenerate form on $\mathfrak{g}$.) Corollary 7.3 can be rephrased as follows.

Corollary 7.4. If $\mathfrak{g}$ is a triangular Lie bialgebra admitting a nondegenerate bilinear form, then

$$
\mathcal{D}(\mathfrak{g}) \cong \mathfrak{g}[t] /\left\langle\left(t^{2}\right)\right\rangle
$$

and the image of the embedding $\mathfrak{g}^{*} \subset \mathcal{D}(\mathfrak{g})$ is

$$
\left(\mathrm{id}+t \sigma^{-1} \circ \gamma\right)\left(\mathfrak{g}_{+}\right) \subset \mathfrak{g}[t] /\left\langle\left(t^{2}\right)\right\rangle
$$

Any complex simple triangular Lie algebra is of the type considered in Corollary 7.4. In this case we recover a result of Stolin, [12]. The proof in [12] relies on works of Berman, Moody and Benkart, Zelmanov on Lie algebras graded by finite root systems. One of the motivations for this work was to show that Corollaries 7.2 and 7.3 follow from the triangularity of the bialgebra structure without assuming simplicity of the algebra.

\section{Acknowledgements}

The authors would like to thank Nicolai Reshetikhin and Sei-Qwon Oh for fruitful discussions.

\section{References}

[1] H. Cartan and S. Eilenberg, Homological algebra, Princeton University press, Princeton, 1957.

[2] V. Chari and A. Pressley, A guide to quantum groups, Cambridge University Press, Cambridge, 1994.

[3] V. G. Drinfeld, On Poisson homogeneous spaces of Poisson-Lie groups, Theoret. and Math. Phys. 95 (1993), 524-525. 
[4] P. J. Hilton and U. Stammbach, A course in homological algebra, Springer, New York, 1997.

[5] G. Hochschild, Group extensions of Lie groups, Ann. of Math. 54 (1951), 96-109.

[6] T. J. Hodges and T. Levasseur, Primitive ideals of $\mathbb{C}_{q}[S L(3)]$, Comm. Math. Phys. 156 (1993), 581-605.

[7] S. L. Levendorskii and Y. S. Soibelman, Algebras of functions on compact quantum groups, Schubert cells, and quantum tori, Comm. Math. Phys. 139 (1991), 141-170.

[8] S. Majid, Foundations of quantum group theory, Cambridge University Press, Cambridge, 1995.

[9] N. Y. Reshetikhin and M. Semenov-Tian-Shansky, Quantum R-matrices and factorization problems, J. Geom. Phys. 5 (1988), 533-550.

[10] M. Semenov-Tian-Shansky, What is a classical r-matrix?, Funct. Anal. Appl. 17 (1983), 259-272.

[11] Dressing transformations and Poisson group actions, Publ. Res. Inst. Math. Sci. 21 (1985), 1237-1260.

[12] A. Stolin, Some remarks on Lie bialgebra structures on simple complex Lie algebras, Comm. Algebra 27 (1999), 4289-4302.

[13] V. S. Varadarajan, Lie Groups, Lie Algebras and Their Representations, SpringerVerlag, New York, 1984.

[14] M. Yakimov, Symplectic leaves of complex reductive Poisson-Lie groups, preprint, math.QA/0004102.

Department of Mathematics, University of Cincinnati, Cincinnati, OH 45221-0025.

E-mail address: timothy.hodges@uc.edu

Department of Mathematics, University of California at Berkeley, Berkeley, CA 94720.

E-mail address: yakimov@math.berkeley.edu 